\title{
A Case Report of Persistent Fifth Aortic Arch Presenting With Severe Left Ventricular Dysfunction
}

\author{
Kouki NaKashima, ${ }^{1}$ MD, Norihiko OKa, ${ }^{1}$ MD, Hidenori Hayashi, ${ }^{1}$ MD, Miyuki Shibata, ${ }^{1}$ MD, \\ Tadashi Kitamura, ${ }^{1}$ MD, Keiichi Itatani, ${ }^{1} \mathrm{MD}$, and Kagami MiYaJi, ${ }^{1} \mathrm{MD}$
}

\begin{abstract}
SUMMARY
According to several previous reports on persistent fifth aortic arch (PFAA), the presentation of the patients was usually either very mild when diagnosed by physical examination including upper body systemic hypertension and systolic murmur, or severe with ductal shock in the neonatal period. In our case, the clinical course was unique with relatively mild narrowing at the distal PFAA and an interrupted fourth aortic arch. It can be classified as medium severity based on the timing of presentation to the hospital. In the present case, severe LV dysfunction suggested sustained narrowing at the junction between the PFAA and the descending aorta and insufficient development of collateral arteries.

We experienced a case with PFAA with severe LV dysfunction. These findings suggest another differential diagnosis for severe LV dysfunction in infancy. (Int Heart J 2014; 55: 87-88)
\end{abstract}

Key words: Interrupted aortic arch

$\mathrm{S}$ ince the first case described by Van Praagh, et al..$^{1)}$ in 1969, several persistent fifth aortic arch (PFAA) cases have been reported, ${ }^{2-5)}$ and PFAA is now considered to be a congenital aortic arch anomaly.

PFAA is usually manifested by physical examination such as hypertension, systolic murmur, and a difference in blood pressure between upper and lower extremities. These symptoms usually appear in the infant or later, when both the distal PFAA and fourth aortic arch become narrow. Because patients diagnosed after the neonatal period commonly have well-developed collateral arteries to the lower body, their symptoms are usually not prominent. On the other hand, a PFAA with severe distal stenosis and an interrupted fourth aortic arch could cause ductal shock in the neonatal period.

We report here a case with PFAA complicated by distal stenosis and an interrupted fourth aortic arch manifested with severe left ventricular (LV) dysfunction at the age of 2 months.

\section{Case Report}

Clinical Summary: A 2-month-old female was admitted to the pediatric intensive care unit due to poor feeding and vomiting and was diagnosed with severe coarctation of the aorta (CoA). Prostaglandin infusion was commenced immediately after the admission. Physical examination revealed a blood pressure difference between the upper and lower extremities. A blood test revealed mildly impaired renal function. An echocardiogram revealed severe LV dysfunction with an ejection fraction (EF) of $10 \%$. Since the blood pressure difference in the upper and lower extremities was ameliorated and the EF and renal function were improved with prostaglandin infusion, the decision was made to perform CoA repair after medical treatment for a few days. Preoperative enhanced computed tomography (CT) was not performed because of her renal dysfunction.

On post admission day 4 , the operation was performed through a left fourth intercostal thoracotomy. Intraoperative findings revealed that the patient had PFAA complicated with an interrupted fourth aortic arch. The distal end of PFAA was severely stenotic, and the ductus arteriosus connected to the distal to the stenotic site was occluded (Figure).

An extended end-to-end anastomosis was performed between the descending aorta and the fourth aortic arch. The PFAA and ductus arteriosus were divided. During crossclamping of the descending aorta, the blood pressure at the lower extremity was well maintained because of the sufficient flow of the collaterals. The postoperative course was uneventful. A postoperative CT showed good flow to the descending aorta from the aortic arch (Figure). The postoperative EF improved gradually up to $50 \%$ on postoperative day 14 .

Comment: According to several previous reports on PFAA, the presentation of the patients was usually either very mild and they were diagnosed by physical examination including systemic hypertension in the upper body and systolic murmur, or severe with ductal shock in the neonatal period. ${ }^{2-5)}$ Different from $\mathrm{CoA}$ or an interrupted aortic arch, the blood flow to the descending aorta is supplied by the ductus arteriosus and the PFAA. This may explain why clinical manifestation is slow in PFAA, and sufficient collateral vessels can develop in the meantime unless the narrowing at the distal PFAA is severe.

From the ${ }^{1}$ Department of Cardiovascular Surgery, Kitasato University School of Medicine, Sagamihara, Japan.

Address for correspondence: Norihiko Oka, MD, Department of Cardiovascular Surgery, Kitasato University School of Medicine, 1-15-1 Kitasato, Minami, Sagamihara, Kanagawa 252-0374, Japan. E-mail: norihiko@rr.iij4u.or.jp

Received for publication June 23, 2013

Revised and accepted August 9, 2013.

Released advance online J-STAGE January 27, 2014.

All rights are reserved to the International Heart Journal Association. 


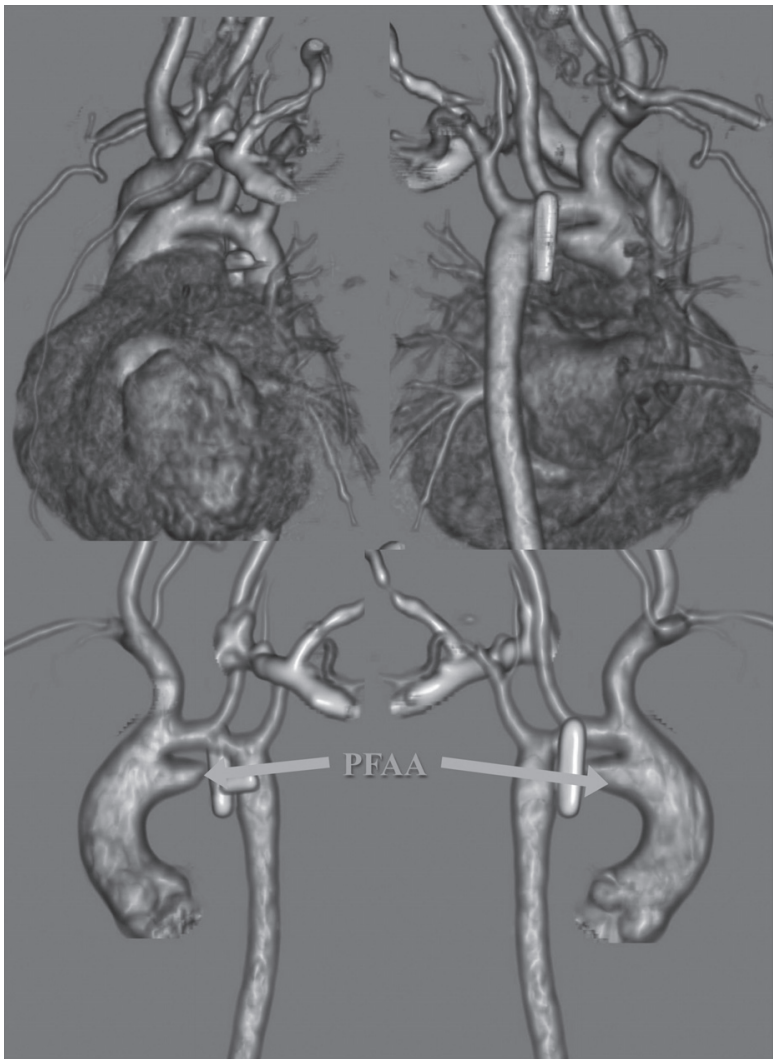

Figure. Images of postoperative computed tomography. Arrows show the PFAA.

Zhao, et al. also proposed that the potential hemodynamic consequences of PFAA seem to be favorable, if the alternative systemic aortic arch flow is sufficient. ${ }^{2}$ In our case, the clinical course was unique with mild narrowing at the distal PFAA and interrupted fourth aortic arch. According to the timing of the hemodynamic collapse, this case can be classified as intermediate type. However, the clinical presentation was serious in this case because of severe LV dysfunction. Severe LV dysfunction was considered to be caused by narrowing at the connection of the PFAA and descending aorta with underdeveloped collateral arteries in our case. It is not clear when the ductus arteriosus closed in this case. As the prostaglandin infusion was effective for the distal stenosis of the PFAA, it can be assumed that the ductus arteriosus, of which occlusion had caused insufficient blood supply to the lower body, was reversibly opened with medical treatment.

In conclusion, we have reported a case with PFAA with severe LV dysfunction. This case suggests that PFAA could be considered to be one of the differential diagnoses for severe LV dysfunction in infancy.

\section{REFERENCES}

1. Van Praagh R, Van Praagh S. Persistent fifth aortic arch in man. Congenital double-lumen aortic arch. Am J Cardiol 1969; 24: 27982.

2. Zhao YH, Su ZK, Liu JF, Cao DF, Ding WX. Surgical treatment of persistent fifth aortic arch associated with interrupted aortic arch. Ann Thorac Surg 2007; 84: 1016-9.

3. Isomatsu Y, Takanashi Y, Terada M, Kasama K. Persistent fifth aortic arch and fourth arch interruption in 28-year-old woman. Pediatr Cardiol 2004; 25: 696-8.

4. Iwase J, Maeda M, Sasaki S, Mizuno A. Images in cardiothracic surgery. Persistent fifth aortic arch. Ann Thorac Surg 2006; 81: 1908.

5. Lambert V, Blaysat G, Sidi D, Lacour-Gayet F. Double-lumen aortic arch by persistent of fifth aortic arch: A new case associated with coarctation. Pediatr Cardiol 1999; 20: 167-9. (Review) 